NISSUNA UMANA INVESTIGAZIONE SI PUO DIMANDARE VERA SCIENZIA S'ESSA NON PASSA PER LE MATEMATICHE DIMOSTRAZIONI LEONARDO DA VINCI

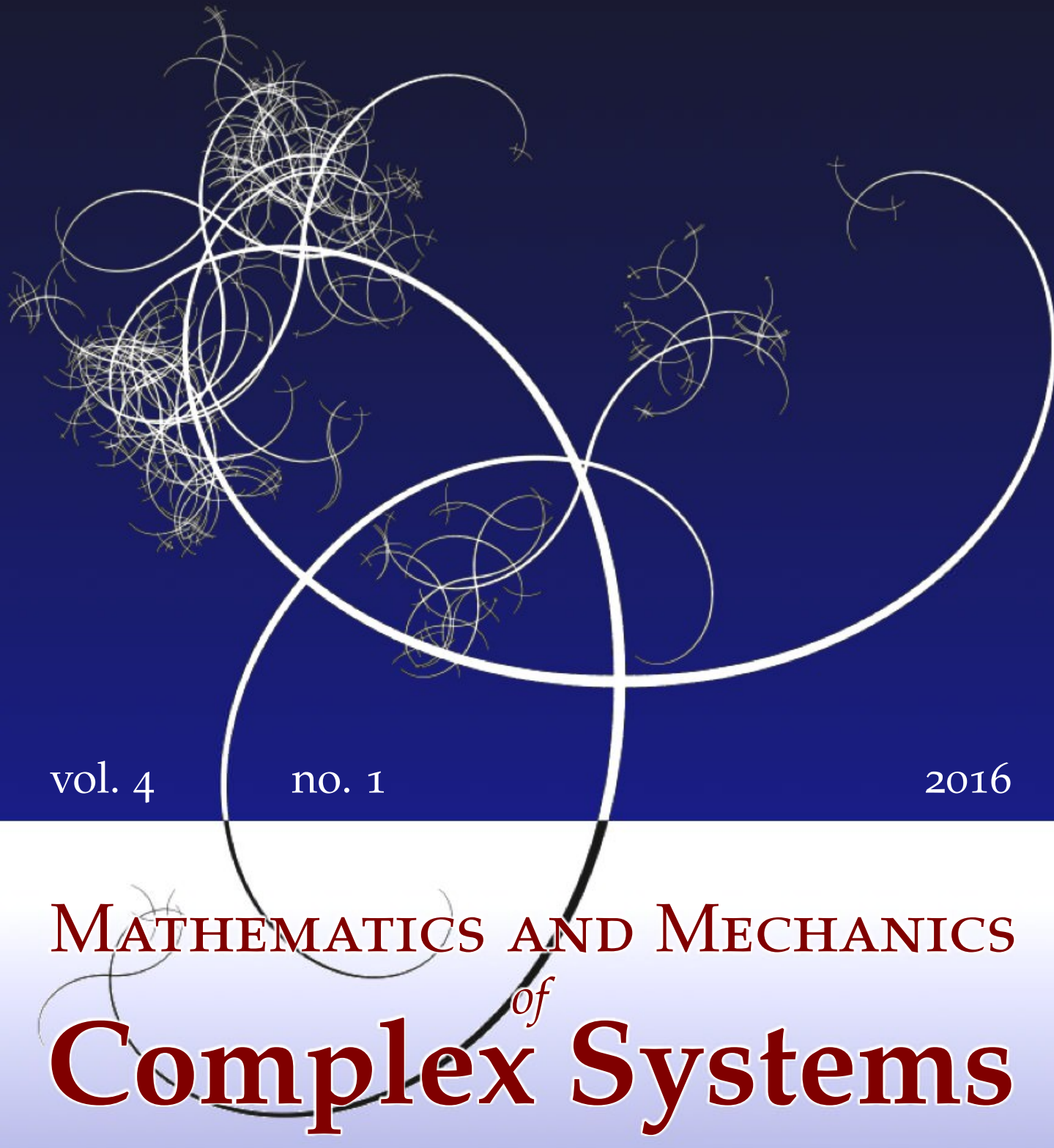

Albrecht BERTRAM AND RAINER GLÜGE GRADIENT MATERIALS WITH INTERNAL CONSTRAINTS 


\title{
GRADIENT MATERIALS WITH INTERNAL CONSTRAINTS
}

\author{
Albrecht Bertram AND RAINER GLÜGE
}

\begin{abstract}
The concept of internal constraints is extended to gradient materials. Here, interesting constraints can be introduced, such as pseudorigid ones. The stresses and the hyperstresses will be given by constitutive equations only up to reactive parts, which do no work during any compatible motion of the body. For the inclusion of thermodynamical effects, the theory is generalized to the case of thermomechanical constraints. Here one obtains reactive parts of the stresses, heat flux, entropy, and energy, which do not contribute to the dissipation. Some critical remarks on the classical concept of internal constraints are finally given. A method to introduce internal constraints in a natural way is described to overcome some conceptual deficiencies of the classical concept.
\end{abstract}

\section{Introduction}

The theory of internal constraints as it is described in, e.g., [Truesdell and Noll 1965, Section 30] is a useful tool for the description of incompressible materials, inextensible composites, and many more material classes. It gives a conceptually sound basis upon which both theoretical and practical investigations can be developed. Particularly, it provides a change of the structure of the basic balance equations, which can be helpful for the construction of solutions of the field problem. This way, the only nonhomogeneous universal solutions for simple materials are those for constrained materials [Ericksen 1955].

On the other hand, there has been an increasing interest in nonclassical extensions of the concept of simple materials. Micromorphic, micropolar, and gradient materials are examples for a blossoming variety of new theories, which go far beyond the classical simple materials. In particular, the inclusion of the second deformation gradient opens the door for many challenging new perspectives for material modeling with the inclusion of internal length scales.

For gradient materials, one wants to introduce internal constraints other than the classical ones to again benefit from such extensions. The question arises whether such an extension is possible, or demands substantial alterations of the entire

\section{Communicated by Francesco dell'Isola.}

MSC2010: 74A30.

Keywords: internal constraints, gradient materials, pseudorigidity. 
format. It turns out, and will be shown in the sequel, that such an extension is in fact straightforward once a theory of gradient materials has been constructed at least within the mechanical context.

The extension to a thermomechanical format is more complicated. This is, however, necessary not only in order to investigate the compatibility of such constraints with the second law of thermodynamics, but also to study the temperature dependence of mechanical constraints.

There has been some discussion about a sound format for the inclusion of the thermodynamical variables into such a theory of internal constraints; see [Green et al. 1970; Trapp 1971; Andreussi and Podio Guidugli 1973; Gurtin and Podio Guidugli 1973; Bertram and Haupt 1976; Casey and Krishnaswamy 1998; Casey 2011; Bertram 2005].

The starting point for the present approach is a suggestion by [Trapp 1971; Bertram 2005], where a rate form of a thermomechanical constraint is assumed and the possibility for reactive parts of the stresses, heat fluxes, and energies is given that are not dissipative during any process that is compatible with the constraint. Again, the extension of this theory to gradient materials is straightforward.

At the end of this contribution, some critical remarks on the standard theory of mechanical constraints are given and a procedure to avoid these shortcomings is suggested.

Notations. Throughout the paper, a dot will denote a scalar product between tensors of arbitrary order. Vectors are denoted by small bold letters like $\mathbf{j}, \mathbf{m}, \mathbf{n}$. Second-order tensors are denoted by capital letters like $\mathbf{J}, \mathbf{S}, \mathbf{T}$, and third-order tensors by $J, G, M$. Since there is not yet a standard notation for odd-order tensors, we have to introduce some operations. The first product we need is the pullback operation of a third-order tensor $\mathbf{G}$ by a second-order tensor $\mathbf{F}$, defined as

$$
\mathbf{F}^{-1} \circ \boldsymbol{G}=\mathbf{F}^{-1} \circ\left(G^{i j k} \mathbf{e}_{i} \otimes \mathbf{e}_{j} \otimes \mathbf{e}_{k}\right):=G^{i j k}\left(\mathbf{F}^{T} \mathbf{e}_{j}\right) \otimes\left(\mathbf{F}^{-1} \mathbf{e}_{j}\right) \otimes\left(\mathbf{F}^{-1} \mathbf{e}_{k}\right) .
$$

Another useful tool is the Rayleigh product between a second-order tensor $\mathbf{F}$ and a tensor of arbitrary order $J$, defined as

$$
\mathbf{F} * J:=J^{i \ldots j}\left(\mathbf{F e}_{j}\right) \otimes \cdots \otimes\left(\mathbf{F e}_{j}\right) .
$$

For orthogonal tensors $\mathbf{F}$ both products coincide.

\section{Simple and gradient materials}

A simple material is defined as one for which the Cauchy stresses are given by a history functional or a process functional of the form

$$
\mathbf{T}\left(\mathbf{x}_{0}, t\right)=\mathscr{F}_{1}\left\{\chi\left(\mathbf{x}_{0}, \tau\right),\left.\operatorname{Grad} \chi\left(\mathbf{x}_{0}, \tau\right)\right|_{\tau=0} ^{t}\right\}
$$


with $\chi$ being the motion of the body in the time interval between some initial time $\tau \equiv 0$ and some final time $t$, and $\mathbf{x}_{0}$ a material point in the reference placement. If we submit this functional to the principle of Euclidean invariance, then we can find reduced forms which identically fulfill this principle. One example for a reduced form is the functional

$$
\mathbf{S}\left(\mathbf{x}_{0}, t\right)=\mathscr{K}_{1}\left\{\left.\mathbf{C}\left(\mathbf{x}_{0}, \tau\right)\right|_{\tau=0} ^{t}\right\},
$$

which assigns to each process in the right Cauchy-Green tensor $\mathbf{C}=\mathbf{F}^{T} \mathbf{F}$ the second Piola-Kirchhoff stress tensor $\mathbf{S}=J \mathbf{F}^{-1} \mathbf{T} \mathbf{F}^{-T}$. Here, $\mathbf{F}$ is the deformation gradient and $J$ its determinant.

If we extend this format to second gradient materials ${ }^{1}$ (see [Bertram 2015a]), the general form of the constitutive functional is

$$
\mathbf{T}\left(\mathbf{x}_{0}, t\right)=\mathscr{F}\left\{\chi\left(\mathbf{x}_{0}, \tau\right), \operatorname{Grad} \chi\left(\mathbf{x}_{0}, \tau\right),\left.\operatorname{Grad} \operatorname{Grad} \chi\left(\mathbf{x}_{0}, \tau\right)\right|_{\tau=0} ^{t}\right\} .
$$

However, for second gradient materials we must expect the existence of a thirdorder hyperstress tensor $\boldsymbol{G}$ for which a second constitutive functional of the form

$$
\boldsymbol{G}\left(\mathbf{x}_{0}, t\right)=\varphi\left\{\chi\left(\mathbf{x}_{0}, \tau\right), \operatorname{Grad} \chi\left(\mathbf{x}_{0}, \tau\right),\left.\operatorname{Grad} \operatorname{Grad} \chi\left(\mathbf{x}_{0}, \tau\right)\right|_{\tau=0} ^{t}\right\}
$$

is needed. Reduced forms of these two functionals are, among others,

$$
\begin{aligned}
& \mathbf{S}\left(\mathbf{x}_{0}, \tau\right)=\mathfrak{k}\left\{\mathbf{C}\left(\mathbf{x}_{0}, \tau\right),\left.\mathcal{K}\left(\mathbf{x}_{0}, \tau\right)\right|_{\tau=0} ^{t}\right\}, \\
& \boldsymbol{H}\left(\mathbf{x}_{0}, \tau\right)=\mathscr{K}\left\{\mathbf{C}\left(\mathbf{x}_{0}, \tau\right),\left.K\left(\mathbf{x}_{0}, \tau\right)\right|_{\tau=0} ^{t}\right\},
\end{aligned}
$$

with the two third-order tensors called the continuity tensor

$$
K:=\mathbf{F}^{-1} \operatorname{Grad} \mathbf{F}
$$

and the material hyperstress tensor

$$
\boldsymbol{H}:=\mathbf{F}^{-1} \circ J \boldsymbol{G}
$$

Both tensors are material tensors and hence invariant under rigid body motions. In [Bertram 2013; 2015a] examples for elastic and plastic materials within such a format are given.

A hyperelastic gradient material would be constituted by an energy function $w(\mathbf{C}, \boldsymbol{K})$ such that the stresses are given by the potential relations

$$
\begin{aligned}
\mathbf{S} & =2 \rho_{0} \partial_{\mathbf{C}} w(\mathbf{C}, \boldsymbol{K}), \\
\mathbf{H} & =\rho_{0} \partial_{\boldsymbol{K}} w(\mathbf{C}, \boldsymbol{K}),
\end{aligned}
$$

with the density in the reference placement $\rho_{0}$.

\footnotetext{
${ }^{1}$ Only these are considered here. Third- and higher-order gradients are beyond the scope of this paper.
} 


\section{Classical internal constraints}

The classical theory of internal constraints after [Truesdell and Noll 1965] and several authors earlier and also later is based on two assumptions.

Assumption 1a (constraint equation for simple materials). There are restrictions upon the possible deformations of the material element such that a scalar-valued function of the deformation gradient $\mathbf{F}$ equals zero for all possible deformations,

$$
\gamma(\mathbf{F})=0 .
$$

Assumption 2a (principle of determinism for simple materials subject to internal constraints). The stress is determined by the history of the deformation only to within an additive part that does no work in any possible motion satisfying the constraint.

Truesdell and Noll [1965] put these assumptions in an axiomatic way, without giving any substantiation for them other than the plausibility of their consequences in particular applications.

If one applies the principle of Euclidean invariance (material objectivity) ${ }^{2}$ to the material function $\gamma$, one can show that a function $\gamma_{\text {red }}(\mathbf{C})$ of the right CauchyGreen tensor $\mathbf{C}=\mathbf{F}^{T} \mathbf{F}$ is objective and, hence, a reduced form.

Such a constraint would be considered as isotropic if it were invariant under arbitrary rotations, that is,

$$
\gamma_{\mathrm{red}}(\mathbf{C})=\gamma_{\mathrm{red}}\left(\mathbf{Q C} \mathbf{Q}^{T}\right)
$$

for all orthogonal tensors $\mathbf{Q}$. In this sense, incompressibility would be an isotropic constraint, while inextensibility in one direction is not isotropic.

By exploiting the second assumption, we start with an additive split of the Cauchy stresses into an extra part, which is determined by a constitutive functional, and a reactive part (for which no constitutive functional exists):

$$
\mathbf{T}=\mathbf{T}_{E}+\mathbf{T}_{R}
$$

so that the specific stress power of the latter,

$$
\frac{1}{\rho} \mathbf{T}_{R} \cdot \mathbf{D}=0
$$

vanishes for every process that is compatible with the constraint, where $\mathbf{D}$ denotes the rate of stretching. We can alternatively express this assumption in terms of a

${ }^{2}$ For a precise introduction of this controversial issue, see [Bertram and Svendsen 2001; Bertram 2005], therein called the principle of invariance under superimposed rigid body motions. 
reactive second Piola-Kirchhoff stress with $\mathbf{S}=\mathbf{S}_{E}+\mathbf{S}_{R}$ :

$$
\frac{1}{\rho_{0}} \mathbf{S}_{R} \cdot \mathbf{C}^{\bullet}=0 \text {. }
$$

If the constraint equation is differentiable, we can bring it into the rate form

$$
\gamma_{\text {red }}(\mathbf{C})^{\bullet}=\partial_{\mathbf{C}} \gamma_{\text {red }}(\mathbf{C}) \cdot \mathbf{C}^{\bullet}=0 .
$$

If we multiply this equation by a Lagrangian multiplier $\alpha$, and subtract it from the constraint equation (17), we find that the second Piola-Kirchhoff reaction stress $\mathbf{S}_{R}$ must have the representation

$$
\mathbf{S}_{R}=\alpha \partial_{\mathbf{C}} \gamma_{\mathrm{red}}(\mathbf{C})
$$

and the Cauchy reaction stress

$$
\mathbf{T}_{R}=\alpha \mathbf{F} \partial_{\mathbf{C}} \gamma_{\text {red }}(\mathbf{C}) \mathbf{F}^{T}
$$

with some scalar field $\alpha$.

As a normalization of the decomposition, we can pose the orthogonality condition

$$
\mathbf{T}_{R} \cdot \mathbf{T}_{E}=0 .
$$

This makes the decomposition unique. However, this is not necessary and often not even practical.

If there is more than one internal constraint (say $N \leq 6$ ), one also has to allow for $N$ reaction stresses, which can be superimposed onto the total stress as

$$
\mathbf{T}=\mathbf{T}_{E}+\sum_{i=1}^{N} \alpha_{i} \mathbf{F} \partial_{\mathbf{C}} \gamma_{\operatorname{red} i}(\mathbf{C}) \mathbf{F}^{T}
$$

with $N$ scalar fields $\alpha_{i}$. In the limit for six independent constraints, $\mathbf{C}$ is completely constrained, the material is rigid, and the stresses are completely reactive. This case will be discussed in the last section of this paper.

\section{Nonclassical internal constraints}

The question arises if one could extend this method to gradient materials. It turns out that such a generalization is straightforward.

It has been shown in [Forest and Sievert 2003; Bertram 2013; 2015a] that the specific stress power for a second gradient material can be brought into the Eulerian and Lagrangian forms

$$
\frac{1}{\rho}(\mathbf{T} \cdot \operatorname{grad} \mathbf{v}+\boldsymbol{G} \cdot \operatorname{grad} \operatorname{grad} \mathbf{v})=\frac{1}{\rho_{0}}\left(\frac{1}{2} \mathbf{S} \cdot \mathbf{C}^{\bullet}+\boldsymbol{H} \cdot \boldsymbol{K}^{\bullet}\right),
$$

respectively. We now generalize our two assumptions to gradient materials. 
Assumption 1b (constraint equation for gradient materials). There are restrictions upon the possible deformations of the material element such that a scalar-valued function of the motion, the deformation gradient, and the second gradient equals zero for all possible deformations

$$
\Gamma(\chi, \operatorname{Grad} \chi, \operatorname{Grad} \operatorname{Grad} \chi)=0 .
$$

If this is understood as a constitutive equation, it must fulfill the Euclidean invariance requirement. This leads to the reduced form of the nonclassical internal constraint

$$
\Gamma_{\text {red }}(\mathbf{C}, K)=0 .
$$

If the constraint equation function is differentiable, we get the rate form of it as

$$
\partial_{\mathbf{C}} \Gamma_{\text {red }} \cdot \mathbf{C}^{\bullet}+\partial_{\boldsymbol{K}} \Gamma_{\text {red }} \cdot \boldsymbol{K}^{\bullet}=0
$$

Assumption 2b (principle of determinism for gradient materials subject to internal constraints). The stresses and the hyperstresses are determined by the deformation process only to within additive parts that do no work in any possible motion satisfying the constraint.

Accordingly, we have the decompositions $\mathbf{T}=\mathbf{T}_{E}+\mathbf{T}_{R}$ and $\boldsymbol{G}=\boldsymbol{G}_{E}+\boldsymbol{G}_{R}$ for the spatial stresses, and $\mathbf{S}=\mathbf{S}_{E}+\mathbf{S}_{R}$ and $\boldsymbol{H}=\boldsymbol{H}_{E}+\boldsymbol{H}_{R}$ for the material ones.

After Assumption 2b, we have

$$
\frac{1}{\rho}\left(\mathbf{T}_{R} \cdot \operatorname{grad} \mathbf{v}+\boldsymbol{G}_{R} \cdot \operatorname{grad} \operatorname{grad} \mathbf{v}\right)=\frac{1}{\rho_{0}}\left(\frac{1}{2} \mathbf{S}_{R} \cdot \mathbf{C}^{\bullet}+\boldsymbol{H}_{R} \cdot \boldsymbol{K}^{\cdot}\right)=0 .
$$

By subtracting an $\alpha$-multiple of the constraint equation in the rate form, we obtain

$$
0=\left[\frac{1}{2 \rho_{0}} \mathbf{S}_{R}-\alpha \partial_{\mathbf{C}} \Gamma_{\text {red }}\right] \cdot \mathbf{C}^{\bullet}+\left[\frac{1}{\rho_{0}} \boldsymbol{H}_{R}-\alpha \partial_{\boldsymbol{K}} \Gamma_{\text {red }}\right] \cdot \boldsymbol{K}^{\bullet},
$$

so that the following equations must hold:

$$
\begin{aligned}
& \mathbf{S}_{R}=\alpha 2 \rho_{0} \partial_{\mathbf{C}} \Gamma_{\text {red }}(\mathbf{C}, \boldsymbol{K}), \\
& \boldsymbol{H}_{R}=\alpha \rho_{0} \partial_{\boldsymbol{K}} \Gamma_{\text {red }}(\mathbf{C}, \boldsymbol{K})
\end{aligned}
$$

with a joint Lagrangian parameter $\alpha$ which couples the two reactive stresses.

As a normalization of the decomposition, one can pose the orthogonality condition

$$
\boldsymbol{H}_{R} \cdot \boldsymbol{H}_{E}+\frac{1}{4} \mathbf{S}_{R} \cdot \mathbf{S}_{E}=0 .
$$

This is, however, not compulsory and perhaps not even practical.

A particular choice of the constraint equation would be to demand that certain components of $K$ must vanish. In such cases the corresponding components of $\mathbf{F}$ must be constant in space. 
For $\mathbf{F}$ having nine independent components and the space having three linear independent directions, $9 \times 3=27$ such constraints on $\operatorname{Grad} \mathbf{F}$ are possible. This, however, reduces to 18 independent constraints because of Schwarz's commutation law since the connection tensor has the right subsymmetry

$$
K_{i j k}=F_{i j, k}=\chi_{i, j k}=\chi_{i, k j}=K_{i k j} .
$$

In [Seppecher et al. 2011] one finds examples of materials with microstructures with such properties.

By imposing 18 independent constraints of this kind, the deformation gradient can only be constant in space. Bodies with this property have been investigated in the past under the label homogeneous strains — see [Sławianowski 1974; 1975] or pseudorigidity - see [Cohen 1981; Cohen and Muncaster 1984; Cohen and MacSithigh 1989; Antman and Marlow 1991; Casey 2004; 2006; 2007] — and critically commented upon by [Steigmann 2006]. However, these approaches are completely different from the present one, since there the homogeneity of strains is imposed on the body as a global constraint, while in the present approach we still assume local constraints as an extension of classical constraints to gradient materials.

\section{Thermomechanical constraints}

Not only in mechanics, but also more general in thermomechanics, the introduction of internal constraints is reasonable. In the literature, several suggestions have been made to generalize the mechanical concepts of constraints to thermodynamics; see [Green et al. 1970; Trapp 1971; Andreussi and Podio Guidugli 1973; Gurtin and Podio Guidugli 1973; Casey and Krishnaswamy 1998; Casey 2011; Bertram 2005]. For the extension of the theory of gradient materials to thermomechanics, see [Bertram 2005; 2015b].

In what follows, we extend the concept introduced in [Trapp 1971; Bertram 2005], where one also finds examples for thermomechanical constraints like temperature-dependent incompressibility or inextensibility.

Assumption 1c (constraint equation for thermomechanical gradient materials). $A$ thermomechanical internal constraint consists of four material functions,

$$
\boldsymbol{J}(\mathbf{C}, \boldsymbol{K}, \theta), \quad \mathbf{J}(\mathbf{C}, \boldsymbol{K}, \theta), \quad \mathbf{j}(\mathbf{C}, \boldsymbol{K}, \theta), \quad j(\mathbf{C}, \boldsymbol{K}, \theta),
$$

of the configuration and the temperature with values in third-order tensors, secondorder tensors, vectors, and scalars, respectively, such that for all admissible thermokinematical processes the constraint equation

$$
\mathbf{J} \cdot \boldsymbol{K}^{\bullet}+\mathbf{J} \cdot \mathbf{C}^{\bullet}+\mathbf{j} \cdot \mathbf{g}_{0}+j \cdot \theta^{\bullet}=0
$$

holds at each instant.

Here, $\theta$ denotes the temperature and $\mathbf{g}_{0}$ is the material temperature gradient. 
Since we made use of material variables, this constraint is already in a reduced form. Note that the first two terms, to which the equation is reduced in the isothermal case, correspond to the mechanical constraint (26) in its rate form, which is therefore included in this format as a special case.

Once again, we have to modify the principle of determinism. There have been several suggestions in the literature whether the reactive parts of the dependent variables shall not produce energy or entropy [Green et al. 1970; Trapp 1971; Gurtin and Podio Guidugli 1973], or neither of the two [Andreussi and Podio Guidugli 1973]. The following assumption is close to what Trapp [1971] suggested and follows [Bertram 2005].

Assumption 2c (principle of determinism for gradient materials with thermomechanical internal constraints). The current values of the hyperstress, stress, heat flux, internal energy, and entropy are determined by the thermokinematical process only up to additive parts that are not dissipative during all admissible processes.

Thus, we have the decompositions of the dependent variables into reactive parts and extra parts:

$\begin{array}{ll}\text { hyperstress } & \boldsymbol{H}=\boldsymbol{H}_{E}+\boldsymbol{H}_{R}, \\ \text { 2nd Piola-Kirchhoff stress } & \mathbf{S}=\mathbf{S}_{E}+\mathbf{S}_{R}, \\ \text { material heat flux } & \mathbf{q}_{0}=\mathbf{q}_{0 E}+\mathbf{q}_{0 R}, \\ \text { internal energy } & \varepsilon=\varepsilon_{E}+\varepsilon_{R}, \\ \text { entropy } & \eta=\eta_{E}+\eta_{R},\end{array}$

and, consequently, also for the

$$
\text { free energy } \psi=\varepsilon_{E}+\varepsilon_{R}-\theta \eta_{E}-\theta \eta_{R}=: \psi_{E}+\psi_{R},
$$

where only the extra terms depend on the thermokinematical process.

The reactive parts shall not be dissipative in the sense of the Clausius-Duhem inequality:

$$
\frac{1}{\rho_{0}}\left(\frac{1}{2} \mathbf{S}_{R} \cdot \mathbf{C}^{\bullet}+\boldsymbol{H}_{R} \cdot \boldsymbol{K}^{\bullet}\right)-\frac{1}{\theta \rho_{0}} \mathbf{q}_{0 R} \cdot \mathbf{g}_{0}-\psi_{R} \cdot-\eta_{R} \theta^{\bullet}=0
$$

for all admissible thermokinematical processes.

If we subtract from this equation an $\alpha$-multiple of the constraint equation (34), we get

$$
\begin{aligned}
\left(\frac{1}{\rho_{0}} \frac{1}{2} \mathbf{S}_{R}-\alpha \mathbf{J}\right) \cdot \mathbf{C}^{\bullet}+\left(\frac{1}{\rho_{0}} \boldsymbol{H}_{R}-\alpha \mathbf{J}\right) \cdot \boldsymbol{K}^{\bullet}-\left(\frac{1}{\theta \rho_{0}} \mathbf{q}_{0 R}+\alpha \mathbf{j}\right) \cdot \mathbf{g}_{0} \\
-\psi_{R}-\left(\eta_{R}+\alpha j\right) \theta^{\bullet}=0
\end{aligned}
$$

for any real $\alpha$. Because of the independence of the terms in brackets of $\mathbf{C}^{\bullet}, K^{\bullet}$, $\mathbf{g}_{0}$ and $\theta^{\bullet}$ in a particular material point, this is solved for all constrained materials 
only by

$$
\begin{aligned}
\boldsymbol{H}_{R} & =\alpha \rho_{0} \mathbf{J}(\mathbf{C}, \boldsymbol{K}, \theta), & & \psi_{R}^{\bullet}=0, \\
\mathbf{S}_{R} & =2 \alpha \rho_{0} \mathbf{J}(\mathbf{C}, \boldsymbol{K}, \theta), & & \eta_{R}=-\alpha j(\mathbf{C}, \boldsymbol{K}, \theta), \\
\mathbf{q}_{0 R} & =-\alpha \rho_{0} \theta \mathbf{J}(\mathbf{C}, \boldsymbol{K}, \theta), & &
\end{aligned}
$$

or, for the spatial versions of the reactive parts,

$$
\begin{array}{lll}
\boldsymbol{G}_{R}=\alpha \rho \mathbf{F} \circ \mathbf{J}(\mathbf{C}, \boldsymbol{K}, \theta) & \text { with } & \boldsymbol{G}_{R}=\mathbf{F} \circ J^{-1} \boldsymbol{H}_{R}, \\
\mathbf{T}_{R}=2 \alpha \rho \mathbf{F} * \mathbf{J}(\mathbf{C}, \boldsymbol{K}, \theta) & \text { with } & \mathbf{T}_{R}=\mathbf{F} * J^{-1} \mathbf{S}_{R}, \\
\mathbf{q}_{R}=-\alpha \rho \theta \mathbf{F} * \mathbf{j}(\mathbf{C}, \boldsymbol{K}, \theta) & \text { with } & \mathbf{q}_{R}=\mathbf{F} * J^{-1} \mathbf{q}_{0 R} .
\end{array}
$$

With this form, for no real $\alpha$ can a contradiction to the Clausius-Duhem inequality occur if the extra terms already fulfill it alone.

As a normalization of the decomposition, one can pose the orthogonality condition

$$
\boldsymbol{H}_{R} \cdot \boldsymbol{H}_{E}+\frac{1}{4} \mathbf{S}_{R} \cdot \mathbf{S}_{E}+\mathbf{q}_{0 R} \cdot \frac{\mathbf{q}_{0 E}}{\theta^{2}}+\rho_{0}^{2} \eta_{R} \cdot \eta_{E}=0
$$

This is, however, not compulsory and perhaps not even practical. As the free energy is only determined up to a constant, we can principally assume $\psi_{R}=0$.

If more than one constraint is active, then the reactive parts are simply additive superpositions of those resulting from each constraint alone.

\section{Critical remarks}

There have been some papers considering the approach of [Truesdell and Noll 1965], like [Bertram 1980; 1982; Podio Guidugli 1990; Antman and Marlow 1991; Carlson and Tortorelli 1996; Carlson et al. 2003], and in their majority they confirm it in the sequel. However, there is one point which needs more attention: the limit of rigidity.

Truesdell and Noll [1965] claim that rigidity is described by $\mathbf{C} \equiv \mathbf{I}$. Since the space of all symmetric tensors is 6-dimensional, rigidity would correspond to 6 independent constraints and the total stress would be completely reactive in this case, since

$$
\mathbf{S}_{R}=\sum_{i=1}^{6} \alpha_{i} \partial_{\mathbf{C}} \gamma_{\operatorname{red} i}(\mathbf{C})
$$

spans the whole space of symmetric second-order tensors. We now consider the case of two simultaneous internal constraints:

(1) (incompressibility)

$$
\gamma_{1}(\mathbf{C})=\operatorname{det} \mathbf{C}-1 \Longrightarrow \mathbf{S}_{R 1}=\alpha_{1} \mathbf{C}^{-1} \text { (a hydrostatic pressure) }
$$




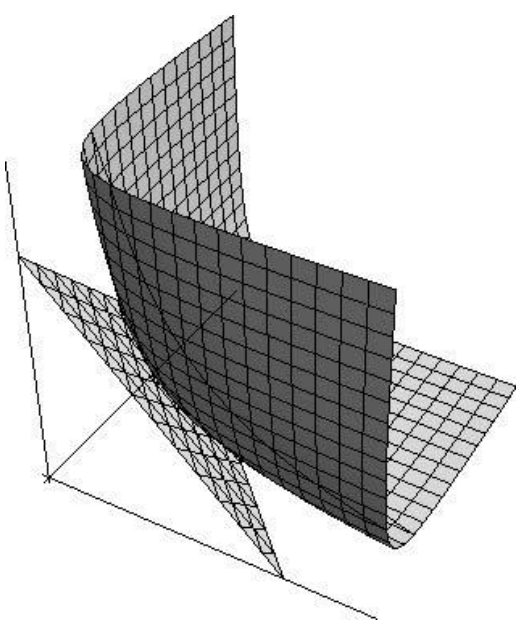

Figure 1. Graphical representation of the two constraint manifolds (43) and (44) in the space of the eigenvalues of $\mathbf{C}$.

(2) (Bell-type $[1973 ; 1985 ; 1996]$ constraint $)^{3}$

$$
\gamma_{2}(\mathbf{C})=\operatorname{tr} \mathbf{C}-3 \Rightarrow \mathbf{S}_{R 2}=\alpha_{2} \mathbf{I} \text {. }
$$

It is not our intention here to comment on the physical significance of this constraint. ${ }^{4}$ Beatty and Hayes [1992] have investigated the geometrical properties of these constraints. ${ }^{5}$ The constraint manifold of $\gamma_{2}$ forms a plane triangle in the space of the eigenvalues of $\mathbf{C}$, while $\gamma_{1}$ forms a curved hypersurface which touches the triangle in only one point; see Figure 1 . So the constraint $\gamma_{2}$ has only one isochoric (or unimodular) point, namely $\mathbf{C} \equiv \mathbf{I}$, which describes rigidity. In this point (and only there) they have a joint tangent plane (that coincides with the plane of $\gamma_{2}$ ). In this point with $\mathbf{C} \equiv \mathbf{I}$, both $\mathbf{S}_{R 1}$ and $\mathbf{S}_{R 2}$ become pressures and $\mathbf{S}_{E}$ is a deviator. However, there is no test possible to identify the constitutive law for this deviator.

An even more absurd example is given by the constraint suggested by Krawietz (personal communication, 2015),

$$
\gamma(\mathbf{C})=(\mathbf{C}-\mathbf{I}) \cdot(\mathbf{C}-\mathbf{I})=0 \Longrightarrow \mathbf{S}_{R}=\mathbf{0},
$$

which also describes rigidity. In this case, the reactive stresses are zero, since $\partial_{\mathbf{C}} \gamma(\mathbf{C})$ vanishes here.

So the format of [Truesdell and Noll 1965] needs some specification of the class of admitted constraint equations to avoid such unphysical results. One has to make sure that they really define a constraint manifold (which is not the case for (45))

\footnotetext{
${ }^{3}$ Bell uses the square root of $\mathbf{C}$, which however does not make much difference here.

${ }^{4}$ See, e.g., [McMeeking 1982; Sellers and Douglas 1990].

${ }^{5}$ See also [Vianello 2014] for the geometry of the constraint manifold.
} 
and, in the case of multiple constraints, that the tangent spaces of these manifolds are independent of each other.

Another remedy would be to introduce the constraints in a natural way, as we show in the next section.

\section{Introduction of internal constraints in a natural way}

In [Bertram 1980; 1982] another approach to establish a theory of internal constraints has been suggested, claiming to be in a natural way. Here, only solids are considered for which the stresses are (at least partly) caused by elastic deformations. The idea there is, roughly speaking, to consider constraint material behavior as a limit of hyperelastic behavior with increasing stiffness for certain deformation modes. If one starts with hyperelastic behavior, one can consider a tangential stiffness tensor with 6 (not necessary different) eigenvalues, called principal stiffnesses in the case of classical (nongradient) materials. If one produces a series of such materials by incrementing one of these eigenvalues to infinity and keeping all others finite, one produces in the limit a material behavior that is constrained in such a way that the deformation mode belonging to this eigenvalue tends to zero if only finite stresses are applied. It has been shown there that for an isotropic or anisotropic hyperelastic material, this construction exactly leads to Assumptions 1a and 2a.

The method to produce internal constraints in a natural way can also be applied to gradient hyperelastic materials. We can linearize the elastic laws (11) and (12) by taking their incremental forms

$$
\begin{aligned}
\mathrm{d} \mathbf{S} & =2 \rho_{0}\left(\partial_{\mathbf{C C}} w(\mathbf{C}, \boldsymbol{K})[\mathrm{d} \mathbf{C}]+\partial_{\mathbf{C} \boldsymbol{K}} w(\mathbf{C}, \boldsymbol{K})[\mathrm{d} \boldsymbol{K}]\right), \\
\mathrm{d} \boldsymbol{G} & =\rho_{0}\left(\partial_{\boldsymbol{K} \mathbf{C}} w(\mathbf{C}, \boldsymbol{K})[\mathrm{d} \mathbf{C}]+\partial_{\boldsymbol{K} K} w(\mathbf{C}, \boldsymbol{K})[\mathrm{d} \boldsymbol{K}]\right),
\end{aligned}
$$

with a

- fourth-order symmetric stiffness tensor (tetradic) $\quad \boldsymbol{C}^{(4)}:=2 \rho_{0} \partial_{\mathbf{C C}} w(\mathbf{C}, \boldsymbol{K})$,

- sixth-order symmetric stiffness tensor (hexadic) $C^{\langle 6\rangle}:=\rho_{0} \partial_{\boldsymbol{K} K} w(\mathbf{C}, \boldsymbol{K})$,

- fifth-order stiffness tensor $C^{\langle 5\rangle}:=2 \rho_{0} \partial_{K \mathbf{C}} w(\mathbf{C}, K)$.

For the linear gradient theory, see [Bertram and Forest 2014]. If we would restrict our attention to central symmetric behavior, the fifth-order stiffness vanishes and the hexadic is known from [Mindlin and Eshel 1968] while the tetradic is the usual one from classical elasticity.

Interesting for us is the stiffness hexadic $C^{\langle 6\rangle}$, since it does not exist for classical materials. We can bring the hexadic into a spectral form,

$$
\boldsymbol{C}^{\langle 6\rangle}=\sum_{i=1}^{18} \lambda_{i} \boldsymbol{P}_{i}^{\langle 6\rangle},
$$


with 18 (not necessarily distinct) eigenvalues $\gamma_{i}$ and the same number of eigenspace projectors of sixth-order, $P_{i}^{\langle 6\rangle}$. These are related to the third-order normalized and orthogonal eigentensors $E_{i}$ of the stiffness hexadic by the sum

$$
\boldsymbol{P}_{i}^{\langle 6\rangle}=\sum_{j=1}^{M_{i}} \boldsymbol{E}_{j} \otimes \boldsymbol{E}_{j}
$$

over the multiplicity $M_{i}$ of the particular eigenvalue. The construction of internal constraints in a natural way consists of taking finite values for all of these eigenvalues except for one, say $\lambda_{1}$.

Let us first consider an eigenvalue of multiplicity one. In the limit, one would not be able to deform the material in the corresponding mode by applying finite stresses. Thus, we obtain the constraint equation (in this case independent of $\boldsymbol{C}$ )

$$
\Gamma(K):=E_{1} \cdot K=0
$$

and expect the reaction hyperstresses after (30) to be

$$
\boldsymbol{H}_{R}=\alpha \partial_{\boldsymbol{K}} \Gamma(\boldsymbol{K})=\alpha \boldsymbol{E}_{1}
$$

with some scalar field $\alpha$.

Such a constraint would be considered as isotropic if it were invariant under arbitrary rotations

$$
\Gamma(\boldsymbol{K})=\Gamma(\mathbf{Q} * \boldsymbol{K})
$$

for all orthogonal tensors $\mathbf{Q}$. Clearly, this is the case if and only if

$$
E_{1}=\mathbf{Q} * E_{1},
$$

i.e., for isotropic tensors. But this would be a rather drastic restriction, which should not be made in general.

We can also superimpose the $M$ constraints of a multiple eigenvalue in one equation:

$$
\Gamma(K):=K \cdots P_{1}^{\langle 6\rangle}[K]=0
$$

Examples and numerical computations of gradient materials with internal constraints will be given in a forthcoming paper by the same authors.

An alternative approach to create internal constraints was suggested by [Casey 1995; Baesu and Casey 2000] in a mechanical setting and [Casey and Krishnaswamy 1998; Casey 2011] in a thermomechanical setting, where the constrained material is identified as an equivalence class of unconstrained ones. 


\section{Example}

We consider the constraint

$$
\operatorname{Grad} J=\mathbf{0} \text {. }
$$

Here, the density of the body can be altered, but only in a homogeneous way in the three spatial directions. One may consider this as a vectorial internal constraint. This may as well be equivalently expressed by a scalar constraint equation by demanding the norm of the vector Grad $J$ be zero.

After the chain rule, we can write $\operatorname{Grad} J$ in terms of $K$ as

$$
\operatorname{Grad} J=J K_{i j k} \mathbf{e}_{k}
$$

with respect to some orthogonal vector basis $\left\{\mathbf{e}_{k}\right\}$. The constraint (55) can be reformulated as

$$
\operatorname{Grad} J \cdot \operatorname{Grad} J / J^{2}=K_{i i k} K_{l l k}=K \cdots P^{\langle 6\rangle}[K]=0
$$

with

$$
\boldsymbol{P}^{\langle 6\rangle}=\mathbf{I} \otimes \mathbf{e}_{j} \otimes \mathbf{I} \otimes \mathbf{e}_{j}
$$

By taking into account the right subsymmetry of the triadic $K$, we can impose the index symmetries between the index pairs $j, k$ and $m, n$ in $P_{i j k l m n}$.

The natural way to introduce this constraint is to add the projector with a penalty parameter to the elasticity hexadic $C^{\langle 6\rangle}$, or likewise to add a penalized term $K_{i i k} K_{l l k}$ to the elastic energy. The latter corresponds to the parameter $a_{2}$ in Mindlin and Eshel's representation [1968, Equation 2.4].

Note added in proof. One can find more results on gradient materials in the Compendium on gradient materials available at http://www.ifme.ovgu.de/ifme_media/ CompendiumGradientMaterialsJan2016.pdf.

\section{Acknowledgment}

The authors want to thank James Casey and Arnold Krawietz for helpful comments on our manuscript.

\section{References}

[Andreussi and Podio Guidugli 1973] F. Andreussi and P. Podio Guidugli, "Thermomechanical constraints in simple materials", Bull. Acad. Pol. Sci. Tech. 21 (1973), 199-205.

[Antman and Marlow 1991] S. S. Antman and R. S. Marlow, "Material constraints, Lagrange multipliers, and compatibility. Applications to rod and shell theories", Arch. Ration. Mech. Anal. 116:3 (1991), 257-299.

[Baesu and Casey 2000] E. Baesu and J. Casey, "A treatment of internally constrained elastic-plastic materials", Int. J. Eng. Sci. 38:15 (2000), 1677-1698. 
[Beatty and Hayes 1992] M. F. Beatty and M. A. Hayes, "Deformations of an elastic, internally constrained material, I: Homogeneous deformations”, J. Elasticity 29:1 (1992), 1-84.

[Bell 1973] J. F. Bell, The experimental foundations of solid mechanics, Handbuch der Physik VIa/1, Springer, Berlin, 1973. Reprinted in Mechanics of Solids I, Springer, Berlin, 1984.

[Bell 1985] J. F. Bell, "Contemporary perspectives in finite strain plasticity", Int. J. Plast. 1:1 (1985), 3-27.

[Bell 1996] J. F. Bell, "The decrease of volume during loading to finite plastic strain”, Meccanica (Milano) 31:5 (1996), 461-472.

[Bertram 1980] A. Bertram, "An introduction of internal constraints in a natural way", Z. Angew. Math. Mech. 60:6 (1980), T100-T101.

[Bertram 1982] A. Bertram, "Material systems-a framework for the description of material behavior”, Arch. Ration. Mech. Anal. 80:2 (1982), 99-133.

[Bertram 2005] A. Bertram, Elasticity and plasticity of large deformations: an introduction, Springer, Heidelberg, 2005.

[Bertram 2013] A. Bertram, "The mechanics and thermodynamics of finite gradient elasticity and plasticity", preprint, Otto-von-Guericke-Universität Magdeburg, 2013, available at http://ovgu.de/ ifme/l-festigkeit/pdf/1/Preprint_Gradientenplasti_finite_16.10.12.pdf.

[Bertram 2015a] A. Bertram, "Finite gradient elasticity and plasticity: a constitutive mechanical framework", Contin. Mech. Therm. 27:6 (2015), 1039-1058.

[Bertram 2015b] A. Bertram, "Finite gradient elasticity and plasticity: a constitutive thermodynamical framework", Contin. Mech. Therm. (online publication March 2015).

[Bertram and Forest 2014] A. Bertram and S. Forest, "The thermodynamics of gradient elastoplasticity”, Contin. Mech. Therm. 26:3 (2014), 269-286.

[Bertram and Haupt 1976] A. Bertram and P. Haupt, "A note on Andreussi-Guidugli's theory of thermomechanical constraints in simple materials", Bull. Acad. Pol. Sci. Tech. 24:1 (1976), 47-51.

[Bertram and Svendsen 2001] A. Bertram and B. Svendsen, "On material objectivity and reduced constitutive equations”, Arch. Mech. Stos. 53:6 (2001), 653-675.

[Carlson and Tortorelli 1996] D. E. Carlson and D. A. Tortorelli, "On hyperelasticity with internal constraints", J. Elasticity 42:1 (1996), 91-98.

[Carlson et al. 2003] D. E. Carlson, E. Fried, and D. A. Tortorelli, "Geometrically-based consequences of internal constraints", J. Elasticity 70:1-3 (2003), 101-109.

[Casey 1995] J. Casey, "Treatment of internally constrained materials", J. Appl. Mech. (ASME) 62:2 (1995), 542-544.

[Casey 2004] J. Casey, "Pseudo-rigid continua: basic theory and a geometrical derivation of Lagrange's equations”, Proc. R. Soc. Lond. A 460:2047 (2004), 2021-2049.

[Casey 2006] J. Casey, “The ideal pseudo-rigid continuum”, Proc. R. Soc. Lond. A 462:2074 (2006), 3185-3195.

[Casey 2007] J. Casey, “A new definition of a pseudo-rigid continuum”, Note Mat. 27:2 (2007), 43-53.

[Casey 2011] J. Casey, "Nonlinear thermoelastic materials with viscosity, and subject to internal constraints: a classical continuum thermodynamics approach", J. Elasticity 104:1-2 (2011), 91104.

[Casey and Krishnaswamy 1998] J. Casey and S. Krishnaswamy, "A characterization of internally constrained thermoelastic materials", Math. Mech. Solids 3:1 (1998), 71-89. 
[Cohen 1981] H. Cohen, "Pseudorigid bodies", Utilitas Math. 20 (1981), 221-247.

[Cohen and MacSithigh 1989] H. Cohen and G. P. MacSithigh, "Plane motions of elastic pseudorigid bodies", J. Elasticity 21:2 (1989), 193-226.

[Cohen and Muncaster 1984] H. Cohen and R. G. Muncaster, "The dynamics of pseudorigid bodies: general structure and exact solutions", J. Elasticity 14:2 (1984), 127-154.

[Ericksen 1955] J. L. Ericksen, "Deformations possible in every compressible, isotropic, perfectly elastic material”, J. Math. Phys. (MIT) 34 (1955), 126-128.

[Forest and Sievert 2003] S. Forest and R. Sievert, "Elastoviscoplastic constitutive frameworks for generalized continua", Acta Mech. 160:1-2 (2003), 71-111.

[Green et al. 1970] A. E. Green, P. M. Naghdi, and J. A. Trapp, "Thermodynamics of a continuum with internal constraints”, Int. J. Eng. Sci. 8:11 (1970), 891-908.

[Gurtin and Podio Guidugli 1973] M. E. Gurtin and P. Podio Guidugli, "The thermodynamics of constrained materials", Arch. Ration. Mech. Anal. 51 (1973), 192-208.

[McMeeking 1982] R. M. McMeeking, "Finite strain tension torsion test of a thin-walled tube of elastic-plastic material”, Int. J. Solids Struct. 18:3 (1982), 199-204.

[Mindlin and Eshel 1968] R. D. Mindlin and N. N. Eshel, "On first strain-gradient theories in linear elasticity”, Int. J. Solids Struct. 4:1 (1968), 109-124.

[Podio Guidugli 1990] P. Podio Guidugli, "Constrained elasticity", Rend. Accad. Naz. Lincei 1:4 (1990), 341-350.

[Sellers and Douglas 1990] H. S. Sellers and A. S. Douglas, "Physical theory of finite plasticity from a theoretical perspective", Int. J. Plast. 6:3 (1990), 329-351.

[Seppecher et al. 2011] P. Seppecher, J.-J. Alibert, and F. Dell'Isola, "Linear elastic trusses leading to continua with exotic mechanical interactions", J. Phys. Conf. Ser. 319:1 (2011), Article ID \#012018.

[Sławianowski 1974] J. J. Sławianowski, "Analytical mechanics of finite homogeneous strains", Arch. Mech. Stos. 26 (1974), 569-587.

[Sławianowski 1975] J. J. Sławianowski, "Newtonian dynamics of homogeneous strains", Arch. Mech. Stos. 27:1 (1975), 93-102.

[Steigmann 2006] D. J. Steigmann, “On pseudo-rigid bodies", Proc. R. Soc. Lond. A 462:2066 (2006), 559-565.

[Trapp 1971] J. A. Trapp, "Reinforced materials with thermo-mechanical constraints", Int. J. Eng. Sci. 9:8 (1971), 757-773.

[Truesdell and Noll 1965] C. A. Truesdell and W. Noll, The non-linear field theories of mechanics, edited by S. Flügge, Handbuch der Physik III/3, Springer, Berlin, 1965. 2nd ed. published in 1992, 3rd ed. edited by S. Antman published in 2004.

[Vianello 2014] M. Vianello, "Internal constraints in finite elasticity: manifolds or not", J. Elasticity 114:2 (2014), 197-211.

Received 16 Jul 2015. Revised 26 Sep 2015. Accepted 8 Nov 2015.

ALBRECHT BERTRAM: albrecht. bertram@ovgu.de

Otto-von-Guericke-Universität Magdeburg, Universitätsplatz 2, D-39106 Magdeburg, Germany

RAINER GLÜGE: gluege@ovgu.de

Otto-von-Guericke-Universität Magdeburg, University of Magdeburg, Universitätsplatz 2,

D-39106 Magdeburg, Germany 